\title{
The association between specific substances of abuse and subcortical intracerebral hemorrhage versus ischemic lacunar infarction
}

\author{
Emma H. Kaplan', Rebecca F. Gottesman ${ }^{2,3}$, Rafael H. Llinas ${ }^{2,3}$ and Elisabeth B. Marsh ${ }^{2,3}$ * \\ ${ }^{1}$ Kennedy-Krieger Institute, Baltimore, MD, USA \\ 2 Department of Neurology, Johns Hopkins University School of Medicine, Baltimore, MD, USA \\ ${ }^{3}$ Department of Neurology, Johns Hopkins Bayview Medical Center, Baltimore, MD, USA
}

\section{Edited by:}

Victor Cruz Urrutia, Johns Hopkins University School of Medicine, USA

Reviewed by:

Steven R. Levine, The State University of New York Health

Science Center at Brooklyn, USA

Bin Jiang, Beijing Neurosurgical

Institute, China

*Correspondence:

Elisabeth B. Marsh, Department of Neurology, Johns Hopkins Hospital, 600 North Wolfe Street, Phipps 446C, Baltimore, MD 21287, USA

e-mail:ebmarsh@jhmi.edu
Background: Hypertension damages small vessels, resulting in both lacunar infarction and subcortical intracerebral hemorrhage (ICH). Substance abuse has also been linked to small vessel pathology. This study explores whether the use of specific substances (e.g., cocaine, tobacco) is associated with subcortical ICH over ischemia in hypertensive individuals.

Methods: Patients with hypertension, admitted with lacunar infarcts (measuring $<2.0 \mathrm{~cm}$ ) or subcortical ICH, were included in analysis. Brain MRIs and head CTs were retrospectively reviewed along with medical records. Demographic information and history of substance use (illicit/controlled: cocaine, heroin, marijuana, benzodiazepines, and methadone; alcohol; and tobacco) was obtained. "Current use" and "history of use" were determined from patient history or a positive toxicology screen. "Heavy use" was defined as: smoking- $\geq 0.5$ packs per day or 10 pack-years; alcohol- average of $>1$ drink per day (women), $>2$ drinks per day (men). Logistic regression was performed with $\mathrm{ICH}$ as the dependent variable comparing those presenting with $\mathrm{ICH}$ to those presenting with ischemia.

Results: Of the 580 patients included in analysis, 217 (37\%) presented with $\mathrm{ICH}$. The average age was similar between the two groups (64.7 versus 66.3 years). Illicit/controlled drug use was associated with a significantly increased risk of $\mathrm{ICH}$ over stroke in unadjusted models ( 25 versus $15 \%, p=0.02$ ), with the largest effect seen in users $\geq 65$ years old (not statistically significant). Smoking was associated with ischemia over $\mathrm{ICH}$ in a dosedependent manner: any history of smoking OR 1.84, Cl 1.19-2.84; current use OR 2.23, Cl 1.37-3.62; heavy use OR 2.48, $\mathrm{Cl} 1.50-4.13$. Alcohol use was not preferentially associated with either outcome $(p=0.29)$.

Conclusion: In hypertensive patients, tobacco use is associated with an increased risk of subcortical ischemia compared to $\mathrm{ICH}$, while use of illicit/controlled substances appears to be predictive of hemorrhage.

Keywords: intracerebral hemorrhage, stroke, hypertension, tobacco, alcohol, drug abuse

\section{INTRODUCTION}

Hypertension is estimated to affect over a third of adults $\geq 20$ years of age in the United States, but fewer than half maintain adequate blood pressure control $(1,2)$. Hypertension is a major risk factor for stroke, and $77 \%$ of first-time strokes occur in hypertensive patients (2). Lacunar infarcts (lacunes), caused by occlusion of a single small artery in a subcortical brain region, account for about a fifth of all strokes (3). Lacunes tend to have a stronger association with hypertension than non-lacunar, cortical strokes (3). Hypertension is also the leading cause of hemorrhagic stroke, particularly involving the subcortical white matter and deep gray structures (4). The majority of studies addressing the impact of other vascular risk factors combine ischemic stroke, intracerebral hemorrhage ( $\mathrm{ICH})$, and subarachnoid hemorrhage without differentiating between stroke subtypes $(2,5)$. Given that $87 \%$ of all strokes are ischemic (2), such data disproportionately represent ischemic infarcts and fail to account for risk factors that may contribute differently to ischemic versus hemorrhagic strokes. Because hypertension is so prevalent and such an important risk factor for both lacunar disease and subcortical ICH $(3,6)$, the identification of other risk factors preferentially leading to one subtype over the other may be useful in individualizing primary prevention strategies.

The relationship between substance abuse and stroke has been studied, though with little differentiation between stroke subtypes. An estimated $9.2 \%$ of adolescents and adults in the United States are current users of illicit/controlled drugs, and an even larger proportion of the population uses legal substances, such as tobacco (22.1\%) and alcohol (52.1\%) (7). Use of any of these substances has been associated with a higher risk of both ICH and ischemic stroke (8-10). 
Our study examines the relationship between substance use and ischemic versus hemorrhagic stroke in hypertensive patients, at higher risk for both. As ICH is typically associated with a poorer long-term prognosis than ischemic stroke $(11,12)$, a better understanding of the specific factors that increase the risk of hemorrhage over ischemia could aid in potential counseling and specific patient-centered risk modification.

\section{MATERIALS AND METHODS STUDY POPULATION}

The Johns Hopkins University School of Medicine Institutional Review Board approved this study. Patient histories, laboratory results, and imaging studies were reviewed retrospectively for 1,980 individuals presenting to the Johns Hopkins Bayview Medical Center with an ischemic stroke or ICH between 2004 and 2012. The following criteria were required for inclusion in further analysis: age $\geq 18$ years, a "history" of hypertension (defined by reported history, use of hypertensive medications, and/or a finding of left ventricular hypertrophy (LVH) on echocardiogram), and subcortical ICH or lacunar infarct on non-contrast head CT or MRI. Patient histories and laboratory results were reviewed to determine demographic information (age, sex, and race), history of hypertension, and history of substance use/abuse (see below).

\section{NEUROIMAGING}

Neuroimaging was retrospectively reviewed by two board certified vascular neurologists for the presence of subcortical disease. We have previously demonstrated excellent inter-rater reliability (kappa 0.76) in evaluating ICH (13). MRI was used in the majority of ischemic stroke workups, while non-contrast CT was used (with or without MRI) in most cases of ICH, and when MRI was not possible (e.g., presence of a pacemaker). A 3-T scanner with a standard quadrature transmit-receive head coil was used for all imaging. T1- and T2-weighted imaging was used to evaluate pathology and to determine the age of any hemorrhage seen on susceptibility-weighted imaging (SWI) (14). Diffusion weighted MRI (DWI) was used to visualize acute infarction.

\section{DEFINING SUBCORTICAL LACUNES AND ICH}

A "symptomatic lacune" was defined as a single, focal, DWI/T2weighted hyperintense lesion measuring $<2.0 \mathrm{~cm}$ (15) in a classic location for lacunar disease (basal ganglia, cerebellum, pons, medulla, midbrain, or subcortical white matter) that corresponded to the acute neurological presentation. Cases of territorial infarcts, where one or more branches of the lenticulostriate arteries were affected due to occlusion of the middle cerebral artery, resulting in small strokes that appeared lacunar, were excluded.

Presence of subcortical ICH was initially determined using non-contrast CT. To be considered subcortical, a hemorrhage had to originate from the subcortical white matter or deep structures. Cortical hemorrhages, defined as those with only cortical and no deep brain involvement, and hemorrhagic conversion of ischemic stroke, were excluded. When necessary, T1- and T2-weighted MRI imaging was used to confirm the origin of hemorrhage (14).

\section{DEFINING SUBSTANCE USE/ABUSE}

The use of both legal (alcohol and tobacco) and illicit/controlled (cocaine, heroin, marijuana, benzodiazepines, and methadone) substances was evaluated. "History" and "current use" of any substance was established using toxicology screens and/or patient admission of prior or current use, respectively. Positive toxicology screen results overrode denials in patient history. "Heavy use" of tobacco was defined as $\geq 0.5$ packs per day or 10 pack-years in men and women. "Heavy use" of alcohol in men was defined as an average of $>2$ drinks per day and in women an average of $>1$ drink per day (16). "Heavy use" could not be defined in illicit/controlled drug use, as dosage information was unavailable.

\section{STATISTICAL ANALYSIS}

Paired $t$-tests (for continuous variables) and Fisher's exact tests (for categorical variables) were used in initial univariate analysis. Multivariable logistic regression was then performed to adjust for confounding demographic factors including age, sex, and race. In multivariable analysis, ICH (versus ischemia) was the dependent variable, while various forms and degrees of substance use/abuse served as the independent variables. All analyses compared those presenting with subcortical ICH to those presenting with lacunar infarction. As a secondary analysis, groups were stratified by age (less than versus greater than or equal to 65 years), and the effect of intensity of use was explored for each substance.

\section{RESULTS}

Of the 1,980 patients initially screened for inclusion in the study, 580 met criteria after review of neuroimaging. Three hundred sixty-three had subcortical lacunar infarcts and the remaining 217 subcortical ICH. Of those excluded, $63 \%$ had cortical pathology and $13 \%$ had no visible lesion. The remaining $24 \%$ were removed from analysis for other reasons (e.g., a cardioembolic source found during work-up). The average age of the included cohort was 65.6 years. Fifty-four percent were female and $27 \%$ were AfricanAmerican. Baseline demographics of the included cohort did not

Table 1 | Patient characteristics.

\begin{tabular}{lcccc}
\hline Demographics & $\begin{array}{l}\text { Total } \\
\text { analyzed } \\
(\boldsymbol{n = 5 8 0 )}\end{array}$ & $\begin{array}{l}\text { Lacunar } \\
\text { infarct } \\
(\boldsymbol{n}=\mathbf{3 6 3})\end{array}$ & $\begin{array}{l}\text { ICH } \\
(\boldsymbol{n}=\mathbf{2 1 7})\end{array}$ & $\boldsymbol{p}$-Value \\
\hline Age (mean year) & 65.6 & 66.3 & 64.7 & 0.28 \\
Sex (\% female) & 54 & 53 & 56 & 0.48 \\
Race (\% African-American) & 27 & 23 & 34 & $\mathbf{0 . 0 1}$ \\
Tobacco (\%) & & & & \\
$\quad$ History & 60.8 & 64.2 & 52.9 & $\mathbf{0 . 0 3}$ \\
$\quad$ Current & 35.5 & 39.7 & 25.9 & $\mathbf{0 . 0 0 5}$ \\
$\quad$ Heavy & 45.6 & 50.6 & 33.0 & $<\mathbf{0 . 0 0 5}$ \\
Alcohol (\%) & 49.5 & 47.8 & 53.3 & 0.30 \\
$\quad$ History & 41.6 & 40.5 & 43.8 & 0.53 \\
$\quad$ Current & 16.8 & 15.9 & 18.8 & 0.48 \\
$\quad$ Heavy & & & & \\
Illicit/controlled substances (\%) & & & & \\
$\quad$ History & 18.2 & 14.8 & 25.0 & $\mathbf{0 . 0 2}$ \\
$\quad$ Current & 14.0 & 11.8 & 18.6 & 0.09 \\
\hline
\end{tabular}

Bold font indicates statistically significant results. 
vary significantly from the entire cohort. Patient characteristics are displayed in Table 1.

\section{SUBSTANCES ASSOCIATED WITH HEMORRHAGE}

Complete results are displayed in Table 1. In univariate analysis, history of illicit/controlled drug use was significantly associated with ICH over ischemia $(p=0.02)$ and current use also trended toward hemorrhage $(p=0.09)$. Smoking was associated with ischemia over ICH (Figure 1). In secondary analysis, the effect appeared dose-dependent: any history of smoking OR 1.84 of smoking over ICH, CI 1.19-2.84; current use OR 2.23 of smoking over ICH, CI 1.37-3.62; heavy use OR 2.48 of smoking over ICH, CI 1.50-4.13. Alcohol was not preferentially associated with either outcome (Figure 1). In multivariate analysis (adjusted for age, race, and sex), in individuals using illicit/controlled drugs the largest effect on increased risk of hemorrhage was seen in individuals age 65 years and greater (OR 1.5 versus 2.5 for current, and 1.7 versus 2.5 for history of use) (Table 2 ), though results were not statistically significant.

\section{DISCUSSION}

Hypertension is prevalent and an important risk factor for both ischemic and hemorrhagic stroke. Specific vascular risk factors may shift the likelihood of one subtype over the other (17). When specifically evaluating substance abuse, tobacco was more strongly associated with ischemia in a dose-dependent manner, while illicit/controlled drug use predicted ICH.

\section{TOBACCO USE VERSUS SUBSTANCE ABUSE - THE DURATION AND INTENSITY OF ABUSE}

The association between tobacco use and ischemic stroke is not surprising. Cigarette smoking has been linked to both small and large vessel atherosclerotic disease and extensively studied in both the cerebrovascular and cardiovascular literature $(10,18,19)$. The relationship between illicit/controlled drug use and hemorrhage is somewhat less intuitive. We posit several potential mechanisms. First and foremost, the population may be different. Individuals who abuse illicit/controlled substances have higher rates of hepatitis C and other chronic illnesses (20), which may result in a relative coagulopathy. On further review of our data, there did not seem to be major differences in the demographic profile between those with hemorrhage versus infarction (17), but we were unable to adjust for all possible medical co-morbidities, including liver disease. A second possibility is that "street drugs" are often cut with agents that may be caustic to blood vessels and result in increased permeability. Furthermore, the use of cocaine results in short, rapid elevations of blood pressure that may cause relatively leaky vessels to rupture, resulting in hemorrhage. While all of these theories may contribute to increased hemorrhage risk, we propose an alternative mechanism related to intensity and duration of use. Those who smoke cigarettes tend to do so consistently (multiple times a day on a daily basis); whereas the use of illicit/controlled substances is typically more episodic, done in a "binge-like" fashion. Continuous damage to vessels through cigarette smoking over time results in thickening of the arteries and plaque buildup, decreasing the diameter of small vessels, and increasing the risk for small vessel ischemia. Due to cost and difficulty of procurement, illicit/controlled substances are often used sporadically.

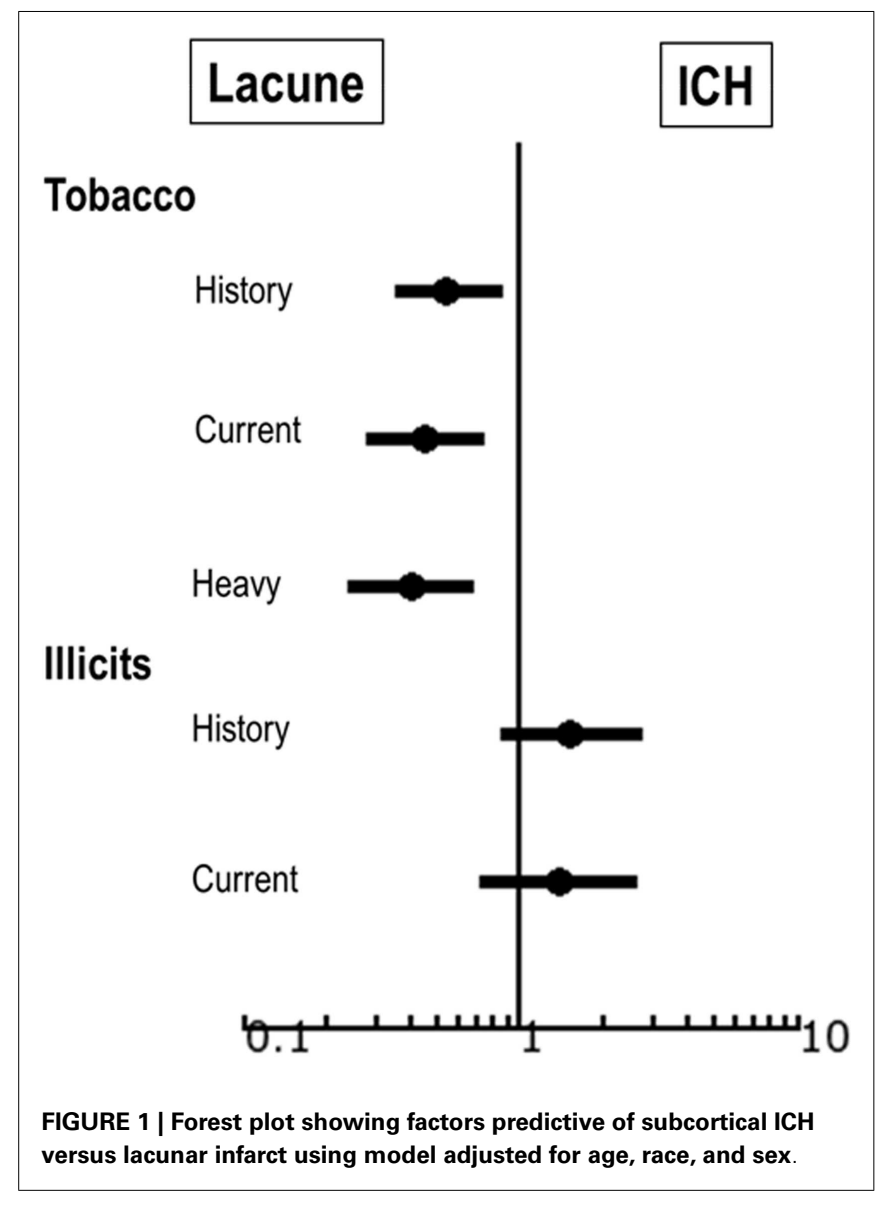

Table 2 | Odds ratios for ICH over lacunar infarct stratified by age

\begin{tabular}{lcc}
\hline Use & OR of ICH over lacune & 95\% Confidence interval \\
\hline $\begin{array}{c}\text { Current } \\
\quad 65 \text { years }\end{array}$ & 1.5 & $0.77-2.85$ \\
$\geq 65$ years & 2.5 & $0.48-12.60$ \\
History & & \\
$\quad<65$ years & 1.7 & $0.94-3.21$ \\
$\geq 65$ years & 2.5 & $0.68-8.91$ \\
\hline
\end{tabular}

Adjusted for age, race, and sex.

Rather than gradually hardening the vessel wall through consistent repetitive damage, this results in vessel injury without thickening or rigidity. In these cases, severe hypertension is more likely to result in friable vessel rupture and subsequent hemorrhage.

The concept that intensity and duration of exposure to a risk factor directly impacts the resulting stroke subtype is consistent with the dose-response relationship we observed with tobacco abuse. Heavier, more frequent use amplifies vessel narrowing without rupture. We have described a similar phenomenon with other vascular risk factors, also dependent on intensity and duration (17). In this study, we also found that the effect of drug use on predicting ICH over ischemia tended to be higher in individuals over the age of 65 , though results were not statistically significant. 
This may be due to longer exposure to other chronic risk factors, such as hypertension, resulting in vessels with significantly abnormal pathology increasing the risk for both stroke subtypes. When exposed to an acute injury in the setting of illicit/controlled drug use, these friable vessels may be even more prone to rupture.

\section{EFFECT OF ALCOHOL USE}

Surprisingly, alcohol use was not preferentially associated with either ischemia or hemorrhage. There are several potential explanations. Studies have shown that moderate levels of alcohol intake increase risk of stroke and cardiac disease, while lower levels appear to be protective (21). This is consistent with our hypothesis that chronic, consistent, moderate to heavy substance use thickens vessel walls over time making thrombosis (ischemia) more likely than rupture $(\mathrm{ICH})$. We did not observe this effect with alcohol in our population. However, we compared ischemia to ICH rather than a control group. Patients who chronically use alcohol are also often malnourished and have relative coagulopathies related to underlying liver disease and/or platelet abnormalities. A coexistent increased risk for hemorrhage may have washed out the effect of increased risk of ischemia. An additional potential explanation is that, given the retrospective nature of the study, alcohol use was poorly recorded. In our experience, compared to both smoking and illicit/controlled drug use, it is a more socially acceptable substance of abuse and often patients will underestimate their true alcohol intake. We relied on patient reports without the ability for further clarification both into overall use and degree of intake. This may have affected the results.

\section{LIMITATIONS}

Our study is not without limitations. It is a retrospective analysis of prospectively collected data, preventing further quantification of illicit/controlled drug use. It is also impossible to ensure that all patients were adequately screened for substance use history, particularly those presenting with aphasia or altered mental status, though all patients admitted to our institution typically undergo screening for substance use as part of their social history. When a patient is unable to provide the history themselves, if available, a family member is asked to provide the information. Additionally, it includes a relatively small number of patients from a single institution. We may therefore be underpowered to show an effect of alcohol or current drug use, or alternatively may have a higher rate of substance use/abuse and underlying co-morbidities than other populations. To that end, there are likely other unmeasured confounders that we were unable to adequately account for, such as hepatitis, which may affect clotting factors. Finally, it is a crosssectional analysis rather than a longitudinal study. An association between substance use and stroke subtype does not necessarily indicate a causal pathway; however, such an association may indicate a potential mechanism that can be further studied. Even with these limitations, our data strongly suggest that the type and degree of substance use increases the risk for certain stroke subtypes.

\section{CONCLUSION}

In hypertensive patients, tobacco use is highly associated with an increased risk of subcortical ischemia compared to ICH in a dose-dependent manner, while use of illicit/controlled substances appears to be predictive of subcortical ICH.

\section{ACKNOWLEDGMENTS}

Elisabeth B. Marsh is supported by a Johns Hopkins School of Medicine Clinician Scientist Award.

\section{REFERENCES}

1. Gillespie CD, Hurvitz KA; Centers for Disease Control and Prevention (CDC). Prevalence of hypertension and controlled hypertension - United States, 20072010. MMWR Surveill Summ (2013) 62:144-8.

2. Roger VL, Go AS, Lloyd-Jones DM, Benjamin EJ, Berry JD, Borden WB, et al. Heart disease and stroke statistics-2012 update: a report from the American Heart Association. Circulation (2012) 125:e2-220. doi:10.1161/CIR. 0b013e31823ac046

3. Jackson C, Sudlow C. Are lacunar strokes really different? A systematic review of differences in risk factor profiles between lacunar and nonlacunar infarcts. Stroke (2005) 36:891-901. doi:10.1161/01.STR.0000157949.34986.30

4. Qureshi AI, Tuhrim S, Broderick JP, Batjer HH, Hondo H, Hanley DF. Spontaneous intracerebral hemorrhage. N Engl J Med (2001) 344:1450-60. doi:10.1056/NEJM200105103441907

5. de Jesús Llibre J, Valhuerdi A, Fernández O, Llibre JC, Porto R, López AM, et al. Prevalence of stroke and associated risk factors in older adults in Havana City and Matanzas Provinces, Cuba (10/66 population-based study). MEDICC Rev (2010) 12:20-6.

6. Manno EM, Atkinson JL, Fulgham JR, Wijdicks EF. Emerging medical and surgical management strategies in the evaluation and treatment of intracerebral hemorrhage. Mayo Clin Proc (2005) 80:420-33. doi:10.4065/80.3.420

7. Substance Abuse and Mental Health Services Administration. Results from the 2012 National Survey on Drug Use and Health: Summary of National Findings, NSDUH Series H-46, HHS Publication No. (SMA) 13-4795. Rockville, MD: Substance Abuse and Mental Health Services Administration (2013).

8. Fonseca AC, Ferro JM. Drug abuse and stroke. Curr Neurol Neurosci Rep (2013) 13:325. doi:10.1007/s11910-012-0325-0

9. Rantakömi SH, Kurl S, Sivenius J, Kauhanen J, Laukkanen JA. The frequency of alcohol consumption is associated with the stroke mortality. Acta Neurol Scand (2014) 130(2):118-24. doi:10.1111/ane.12243

10. Wolf PA, D’Agostino RB, Kannel WB, Bonita R, Belanger AJ. Cigarette smoking as a risk factor for stroke: the Framingham study. JAMA (1988) 259:1025-9. doi:10.1001/jama.259.7.1025

11. Andersen KK, Olsen TS, Dehlendorff C, Kammersgaard LP. Hemorrhagic and ischemic strokes compared: stroke severity, mortality, and risk factors. Stroke (2009) 40:2068-72. doi:10.1161/STROKEAHA.108.540112

12. Lee WC, Joshi AV, Wang Q, Pashos CL, Christensen MC. Morbidity and mortality among elderly Americans with different stroke subtypes. Adv Ther (2007) 24:258-68. doi:10.1007/BF02849893

13. Marsh EB, Gottesman RF, Hillis AE, Urrutia VC, Llinas RH. Serum creatinine may indicate risk of symptomatic intracranial hemorrhage after iv TPA. Medicine (2013) 92:317-23. doi:10.1097/MD.0000000000000006

14. Bradley WG. MR appearance of hemorrhage in the brain. Radiology (1993) 189:15-26. doi:10.1148/radiology.189.1.8372185

15. The SPS3 Investigators. Effects of clopidogrel added to aspirin in patients with recent lacunar stroke. N Engl J Med (2012) 367:817-25. doi:10.1056/ NEJMoa1204133

16. U.S. Department of Agriculture and U.S. Department of Health and Human Services. Dietary Guidelines for Americans. 7th ed. Washington, DC: US Government Printing Office (2010). p. 30-2.

17. Marsh EB, Gottesman RF, Hillis AE, Maygers J, Lawrence E, Llinas RH. Predicting symptomatic intracerebral hemorrhage versus lacunar disease in patients with longstanding hypertension. Stroke (2014) 45:1679-83. doi:10. 1161/STROKEAHA.114.005331

18. Colditz GA, Bonita R, Stampfer MJ, Willett WC, Rosner B, Speizer FE. Cigarette smoking and risk of stroke in middle-aged women. N Engl J Med (1988) 318:937-41. doi:10.1056/NEJM198804143181501

19. Kelly TN, Gu D, Chen J, Huang JF, Chen JC, Duan X. Cigarette smoking and risk of stroke in the Chinese adult population. Stroke (2008) 39:1688-93. doi:10.1161/STROKEAHA.107.505305

20. Alter MJ, Kruszon-Moran D, Nainan OV, McQuillan GM, Gao F, Moyer LA. The prevalence of hepatitis c virus infection in the United States, 1988-1994. N Engl J Med (1999) 341:556-62. doi:10.1056/NEJM199908193410802 
21. Mukamal KJ, Ascherio A, Mittleman MA, Conigrave KM, Camargo CA, Kawachi I. Alcohol and risk for ischemic stroke in men: the role of drinking patterns ad usual beverage. Ann Intern Med (2005) 142:11-9. doi:10.7326/0003-4819-1421-200501040-00007

Conflict of Interest Statement: The authors declare that the research was conducted in the absence of any commercial or financial relationships that could be construed as a potential conflict of interest.

Received: 26 June 2014; accepted: 27 August 2014; published online: 10 September 2014.
Citation: Kaplan EH, Gottesman RF, Llinas RH and Marsh EB (2014) The association between specific substances of abuse and subcortical intracerebral hemorrhage versus ischemic lacunar infarction. Front. Neurol. 5:174. doi: 10.3389/fneur.2014.00174 This article was submitted to Stroke, a section of the journal Frontiers in Neurology. Copyright (C) 2014 Kaplan, Gottesman, Llinas and Marsh. This is an open-access article distributed under the terms of the Creative Commons Attribution License (CC $B Y)$. The use, distribution or reproduction in other forums is permitted, provided the original author(s) or licensor are credited and that the original publication in this journal is cited, in accordance with accepted academic practice. No use, distribution or reproduction is permitted which does not comply with these terms. 